\title{
Stratification of SNOT-22 scores into mild, moderate or severe and relationship with other subjective instruments*
}

\author{
Shamim Toma', Claire Hopkins² \\ ' ENT Dept, Kennington Road, Willesborough, Ashford, Kent, United Kingdom \\ ENT Dept, Guy's and St Thomas' Hospitals NHS Trust, London, United Kingdom
}

Rhinology 54: 129-133, 2016

DOI:10.4193/Rhino15.072

${ }^{*}$ Received for publication:

March 20, 2015

Accepted: November 27, 2015

\begin{abstract}
\section{Aims and objectives}

The European Position Paper on Rhinosinusitis and Nasal Polyps provides treatment algorithms based on the mild/moderate/ severe (MMS) classification. To date there has been no statistically validated stratification of the SNOT-22 score according to this classification.
\end{abstract}

\section{Methods}

65 consecutive patients diagnosed with CRS completed a SNOT-22, VAS and rated their symptoms according to MMS and impact on quality of life.

\section{Results}

The median SNOT 22 scores varied between the 3 MMS categories. The interquartile ranges for the respective MMS groups were: Mild 8-17, Moderate 22.5-48, Severe 54-83. Median values for the respective MMs groups were: Mild 12, Moderate 36 and Severe 66. $15.38 \%$ of patients in the 'mild' category, $95.24 \%$ in the 'moderate' category and $100 \%$ in the 'severe' category feel their QoL is affected. There was a strongly positive correlation between the SNOT-22 and VAS scores.

\section{Conclusion}

We propose a statistically validated definition for stratification of the SNOT-22, with 'Mild' being defined on the SNOT-22 score as 8-20 inclusive, 'moderate' as $>20-50$ and 'severe' as $>50$.

Key words: chronic rhinosinusitis, patient rated outcome meansure, endoscopic sinus surgery, disease severity

\section{Introduction}

Patient reported outcome measures (PROMS), which assess the outcomes of care delivered from a patient's perspective are becoming increasingly important

The Global Allergy and Asthma Network of Excellence (GA2LEN) identified a high prevalence of chronic rhinosinusitis (CRS), around $11 \%$ in adult Europeans ${ }^{(1)}$. CRS results in a considerable burden both on individuals in terms of reduced quality of life (2) and activity, work, sleep and social limitations, and on health care systems in terms of cost and resources ${ }^{(3)}$. The primary aim of the treatment of CRS is reducing the impact of symptoms on the quality of life of the patient. Therefore, effective and successful treatment of CRS has an increasing clinical and economic importance. The move towards greater transparency within healthcare and increasing involvement of patient's choices about their care, the use of measures of health-related quality of life (HRQOL) to evaluate medical and surgical treatment will increase.

A variety of QoL instruments have been developed and validated in order to classify severity of disease and the subjective 
outcome of patients to treatments. Stratification of disease severity facilitates guideline development, allowing patients to be categorised by symptom severity. Lim et al. ${ }^{(4)}$ defined the relationship between mild/moderate/severe (MMS) and a visual analogue scale (VAS) patient-classification rated measure of severity for CRS: 'mild' as being 0-3 inclusive, 'moderate', as >3-7 inclusive and 'severe' as >7-10 inclusive. Current treatment algorithms defined by the European Position Paper on Rhinosinusitis and Nasal Polyps (EPOS) are based on this MMS classification ${ }^{(5)}$. EPOS guidelines do not currently include stratification of disease severity by other instruments.

Disease specific PROMs readily identify the most important symptoms to the patient, quantify the severity of all commonly associated symptoms and home in on key aspects with the consultation in addition to recording clinical progress. They aid identifying the aims of treatment and are more likely to be sensitive to clinically relevant changes than generic outcome measures.

The 22-item Sinonasal Outcome Test (SNOT-22) is a disease specific, validated, patient rated outcome measure ${ }^{(6)}$. It is also considered the most suitable tool in terms of ease of use ${ }^{(7)}$. It contains the key diagnostic symptoms included in the EPOS definition for CRS, as well as other items of importance to patients with CRS. It is being increasingly used to routinely measure disease specific quality of life in clinical practice.

In order to facilitate application of the EPOS guidelines to patients based on their SNOT-22 scire, we set out to stratify patients using their SNOT-22 scores according to the mild/ moderate/ severe (MMS) classification. We also wanted to evaluate the relationship between the SNOT-22 score and VAS score to ensure that they are measuring the same disease construct, and with overall quality of life.

\section{Materials and methods}

\section{Study population}

65 consecutive patients attending clinic for treatment of chronic rhinosinusitis (defined by the EPOS Criteria) participated. Subjects were asked to fill in a questionnaire in which they (a) completed a SNOT-22, (b) rated their overall symptoms of CRS on a $10 \mathrm{~cm}$ VAS scale, in answer to the question "How troublesome are your symptoms of rhinosinusitis", (c) self-categorised their overall symptoms as 'mild', 'moderate' or 'severe' (d) indicated whether their symptoms affected the quality of their life.

\section{Statistical analysis}

Data were collected using Microsoft Excel 2009 (Microsoft, Redmond, WA, US) and the statistical analyses performed in STATA. The relationship between (a) SNOT 22 scores and the patients'

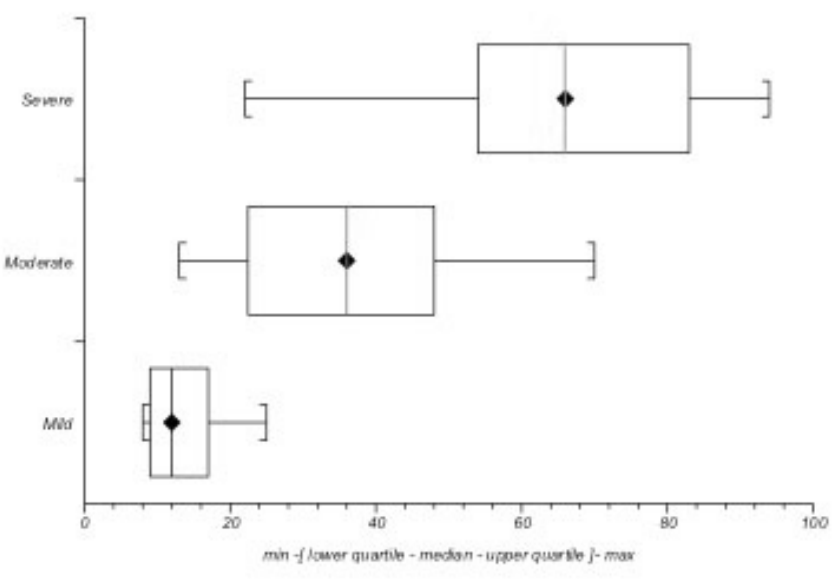

Figure 1. Box and whisker plot SNOT-22 scores for Mild, Moderate and Severe.

self-rated MMS classification and (b) SNOT 22 scores and QoL were examined using box plots showing the median values and the upper and lower quartiles. Receiver Operating Characteristic (ROC) curve was employed in further analysing the relationship between SNOT 22 and QoL.

The correlation between SNOT-22 and VAS scores was examined using Spearman's Rho Correlation Coefficient.

\section{Results}

Demographics and overall results

Of the 65 patients analysed 35 were male and 30 were female.

The age ranged from 26 to 79 and the mean age was 39 .

Thirteen patients rated their symptoms as mild, 21 as moderate and 31 as severe. The VAS scores ranged from 0 to 10, with a mean VAS score of 5.1. The SNOT 22 scores ranged from 4 to 94 , with a mean SNOT 22 score of 46.3 . A total of 52 subjects described their symptoms as affecting their quality of life.

\section{SNOT-22 scores and MMS}

As expected, the median SNOT 22 scores varied between the 3 MMS categories. Table 1 and Figure 1 illustrate the median value, upper/ lower quartiles and extreme values of the SNOT 22 scores

Table 1. MMS and SNOT 22 scores.

\begin{tabular}{|lccc|}
\hline SNOT 22 scores & Mild & Moderate & Severe \\
\hline Lower extreme & 8 & 13 & 22 \\
\hline 25th percentile & 8 & 22.5 & 54 \\
\hline Median & 12 & 36 & 66 \\
\hline 75th percentile & 17 & 48 & 83 \\
\hline Upper extreme & 25 & 70 & 94 \\
\hline
\end{tabular}




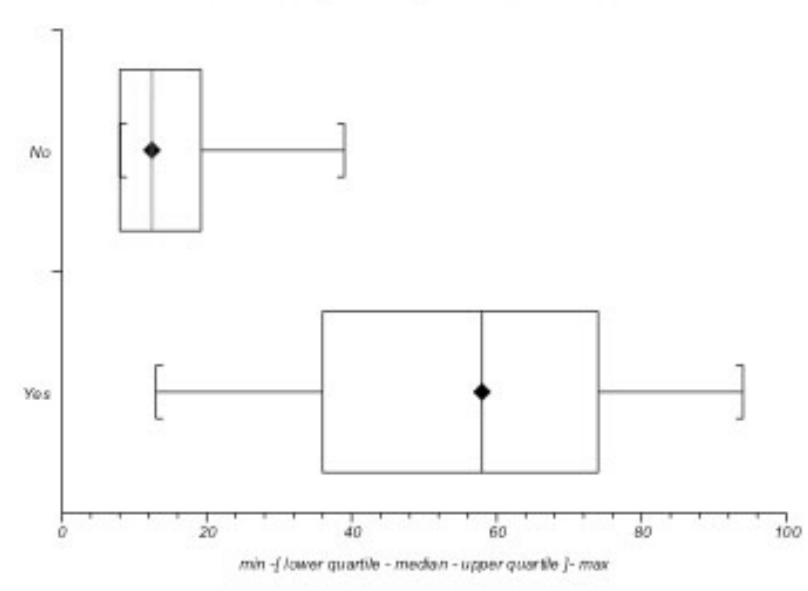

Figure 2. Box and whisker plot for SNOT-22 scores and impairment of quality of life.

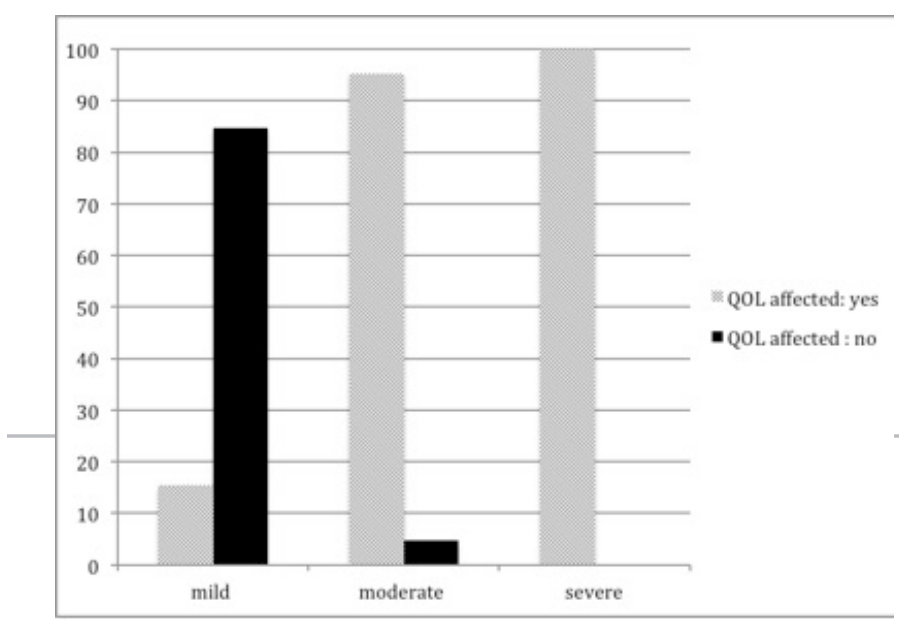

Figure 3. Percentage of subjects reporting that QoL is affected in each MMS category. for the three categories of MMS.

The interquartile ranges for the respective MMS groups were: Mild 8-17, Moderate 22.5-48, Severe 54-83. Median values for the respective MMs groups were: Mild 12, Moderate 36 and Severe 66.

\section{SNOT-22 scores and QoL}

For the group in which QoL scores were affected the reported SNOT-22 scores had a median score of 54 and an interquartile range of 32.5- 72.5. The group of subjects in which QoL was not affected the reported SNOT-22 scores had a median of 12.5 and an interquatile range of 8.5-21.25. Figure 2 illustrates the median value, upper/ lower quartiles and extreme values of the SNOT 22 scores by impairment of quality of life.

\section{MMS and QoL}

Two out of 13 subjects in the 'mild' category felt their quality of life was also affected. Quality of life was affected in 20 out of 21 in the 'moderate group' and all 31 patients in the 'severe' group (Figure 3).

\section{SNOT-22 and VAS}

There was a strongly positive correlation between the SNOT-22 and VAS scores (Rho $=0.84913, \mathrm{p}<0.001$ ). A scatterplot depicts the results in Figure 4.

\section{Analysis}

SNOT 22 scores and MMS

Based on the interquartile results of our study and employing multiples of ten only the following definition is proposed: 'Mild' being defined on the SNOT-22 score as 8-20 inclusive, 'moderate' as $>20-50$ and 'severe' as $>50$. A score of less than 8 has previously been shown to be consistent with that of the normal population, and the results presented herein.

Using this definition means that $11 / 13=84.6 \%$ patients in our study with a score of 0-20 also classified their symptoms as 'mild', $17 / 21=80.9 \%$ of patients with a score of $>20-50$ classified their score as 'moderate' and $24 / 31=77.4 \%$ of subjects with a score of $>50$ classified their score as 'severe'.

\section{SNOT 22 scores and QoL}

In examining the relationship between QoL and SNOT score, ROC analysis shows that SNOT 22 scoring is a good discriminator of whether QoL is affected (area under the curve $0.94295 \% \mathrm{Cl}$ 0.881-1.00) (Figure 5). When the SNOT-22 was dichotomised, a score above 20 (ie moderate/severe) has a sensitivity of $\mathrm{x} \%$ and specificity of $y \%$ in predicting impaired quality of life.

\section{MMS and QoL}

Examining the relationship between MMS categorisation and QoL reveals that $15.38 \%$ of patients in the 'mild' category, $95.24 \%$ in the 'moderate' category and $100 \%$ in the 'severe' category feel their QoL is affected. Dichotomising by mild versus moderate/severe has a sensitivity of $98.1 \%$ and specificity of $81.8 \%$ in predicting impaired quality of life, a positive predictive value of $96.2 \%$ and a negative predictive value of $91.7 \%$.

\section{Discussion}

PROMs are increasingly being used in clinical practice. The SNOT-22 has been used in over 200 published studies and is translated into several common languages. Although a greater respondent burden than an MMS grading or VAS score, it may still be completed in under 5 minutes. As it contains the four diagnostic symptoms included in the EPOS definition of CRS, and additional to broader symptoms across all important domains, it forms a useful part of the clinical record. A number 


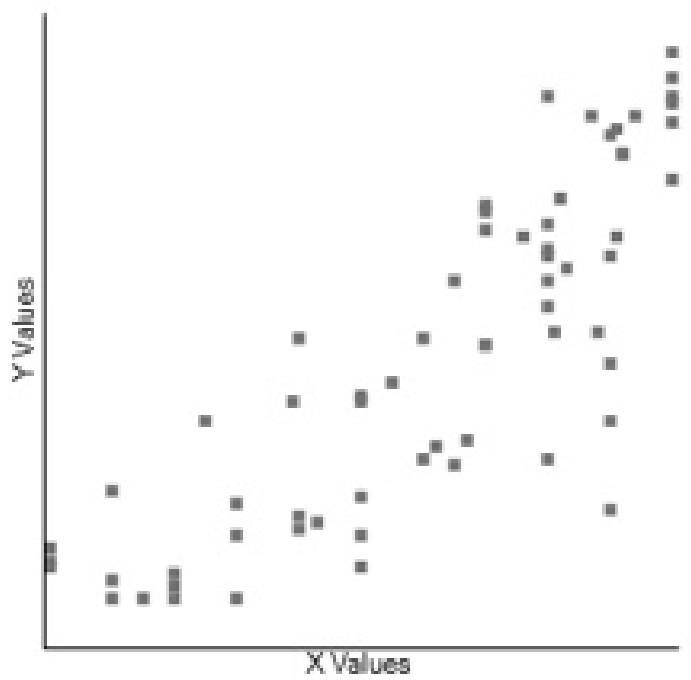

Figure 4. Spearman's Rho Coefficient for SNOT-22 scores (y axis) and VAS scores ( $x$ axis).

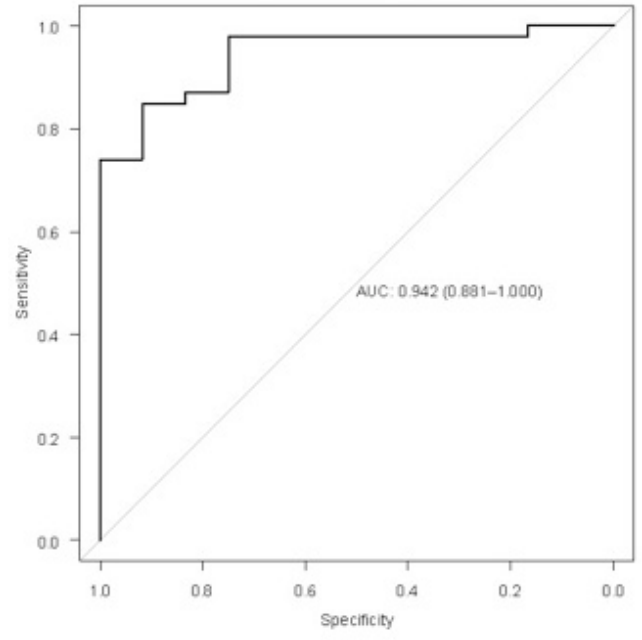

Figure 5. ROC curve for QOL affected as predicted by SNOT 22 score. of papers have arbitrarily categorised SNOT-22 scores according to mild, moderate and severe disease. However, to date no validated stratification of the SNOT-22 has been published. Our results now provide this classification, with 'Mild' being defined on the SNOT-22 score as 8-20 inclusive, 'moderate' as >20-50 and 'severe' as $>50$.

This classification will facilitate the use of the SNOT-22 in conjunction with treatment algorithms described by EPOS, where patient pathways are determined by disease severity into mild moderate and severe. This will reduce the need for both measures to be recorded. It also facilitates classification of patients for research purposes.

The very strong correlation between both the SNOT-22 and VAS score in CRS confirms that these tools measure the same disease construct, and therefore either is suitable for subclassification.

It must be remembered that in this validation we are applying population means to define symptom severity according to mild, moderate and severe. However it has been shown previously that the correlation between symptom severity measured with the SNOT-22 and measures of disease severity such the Lund-Mackay score is poor ${ }^{(8)}$, as there are many internal patient factors (e.g.gender, age) and external environmental factors (e.g. social support, cultural expectations) that modify the impact of disease on quality of life. Thus while dichotomising to mild versus moderate/severe has both high specificity and sensitivity for predicting impaired quality of life, and small number of patients with both mild symptoms or mild disease burden may report a high impact of disease.

\section{Conclusion}

We propose a statistically validated definition for stratification of the SNOT-22, with 'Mild' being defined on the SNOT-22 score as 8-20 inclusive, 'moderate' as $>20-50$ and 'severe' as $>50$.

\section{Acknowledgement}

We would like to thank Prof Mark Haggard for his statistical advice.

\section{Authorship contribution}

ST: data collection and analysis, drafting manuscript; $\mathrm{CH}$ : study design, data collection and analysis, revision of manuscript.

\section{Conflict of interest}

Consultancy work: Acclarent.

No financial disclosures.

\section{References}

1. Hastan D, Fokkens WJ, Bachert C, Newson RB, Bislimovska J, Bockelbrink A, et al. Chronic rhinosinusitis in Europe--an underestimated disease. A GA(2)LEN study. Allergy. 2011 Sep;66(9):1216-23.
2. Gliklich RE, Metson R. The health impact of chronic sinusitis in patients seeking otolaryngologic care. Otolaryngol Head Neck Surg. 1995 Jul;113(1):104-9.

3. Bhattacharyya N. The economic burden and symptom manifestations of chronic rhinosinusitis. Am J Rhinol. 2003 JanFeb; 17(1):27-32

4. Lim M, Lew-Gor S, Darby $Y$, Brookes N Scadding G, Lund VJ. The relationship between subjective assessment instruments in chronic rhinosinusitis. Rhinology. 
2007 Jun;45(2):144-7.

5. Fokkens WJ, Lund VJ, Mullol J, Bachert C, Alobid I, Baroody F, et al. European Position Paper on Rhinosinusitis and Nasal Polyps 2012. Rhinol Suppl. $2012 \operatorname{Mar}(23): 3$ p preceding table of contents, 1-298.

6. Hopkins C, Gillett S, Slack R, Lund VJ Browne JP. Psychometric validity of the 22-item Sinonasal Outcome Test. Clin Otolaryngol. 2009 Oct;34(5):447-54.

7. Morley AD, Sharp HR. A review of sinonasa outcome scoring systems - which is best? Clin Otolaryngol. 2006 Apr;31(2):103-9.

8. Hopkins C, Browne JP, Slack R, Lund V, Brown P. The Lund-Mackay staging system for chronic rhinosinusitis: how is it used and what does it predict? Otolaryngol Head Neck Surg 2007;137(4):555-61.

Miss Claire Hopkins FRCS (ORLHNS)

DM

ENT Dept

Guy's and St Thomas' Hospitals

NHS Trust

London SE1 9RT

United Kingdom

Tel: +44-207-188 2215

E-mail: Clairehopkins@yahoo.com 